\title{
Goldstone modes in vacuum decay and first-order phase transitions
}

\author{
N J Günther $\dagger \&$, D A Nicole $\ddagger$ and D J Wallace $\nmid \S$ \\ $\uparrow$ Department of Physics, The University, Southampton S09 5NH, UK \\ $\uplus$ Department of Physics and Astronomy, University of Pittsburgh, Pittsburgh, Pa 15260 , \\ USA
}

Received 27 April 1979

\begin{abstract}
We introduce effective Hamiltonians for Goldstone modes of the Euclidean group, representing fluctuations in the surface of a critical droplet or in the interface between two phases. The Euclidean invariance is non-linearly realised on the Goldstone fields. The Hamiltonians are non-renormalisable in more than one dimension, showing that the disappearance of a phase transition in one dimension for systems with a discrete symmetry may be interpreted in terms of the infrared instabilities induced by these modes. The existence and form of these Hamiltonians indicates the universality of the essential singularity at a first-order phase transition in models with Euclidean invariance.
\end{abstract}

\section{Introduction}

The importance of Goldstone modes for systems with a continuous symmetry is well known. In the context of phase transitions one thinks conventionally in terms of a Heisenberg-type model with $\mathrm{O}(n)$ symmetry. Below the critical temperature $T_{\mathrm{c}}$ there are $(n-1)$ Goldstone modes whose energy goes to zero in the limits of long wavelength and zero external field $H$. Because of these Goldstone modes, the limit $H \rightarrow 0$ may be thought of as a critical point for all $T<T_{c}$. It becomes important to identify the effective Hamiltonian describing the interactions amongst the critical modes alone. In terms of an isotropic ferromagnet, the effective Hamiltonian is the spin-wave theory, which was used by Holstein and Primakoff (1940) to predict a singular term $H^{-1 / 2}$ in the longitudinal susceptibility in three dimensions. More generally, the effective Hamiltonian is invariant under the full $\mathrm{O}(n)$ symmetry group realised in a non-linear way on the $(n-1)$ Goldstone fields. These so-called non-linear $\sigma$ models control the singularities induced by the Goldstone modes below $T_{c}$. In particular, in $d$ dimensions they imply a term $H^{(d-4) / 2}$ in the longitudinal susceptibility which exists up to the critical temperature $T_{\mathrm{c}}$ (K G Wilson unpublished, Wallace and Zia 1975, Horner and Schäfer 1978 and references therein). Furthermore, this singularity shows that, in two dimensions, fluctuations in the longitudinal field become as singular as those in the Goldstone fields themselves. This is consistent with the theorem that the spontaneous magnetisation is zero at all temperatures in two dimensions for such systems (Mermin and Wagner 1966, Hohenberg 1967, Coleman 1973); the Goldstone modes induce

\$ Permanent address from 1.10.79: Department of Physics, The University of Edinburgh, EH9 3JZ, UK. 
infrared instabilities which destroy the ordered phase. The special role of two dimensions is correspondingly reflected in the ultraviolet properties of these non-linear Hamiltonians; they are non-renormalisable in more than two dimensions and just renormalisable in two. This property has been exploited (Polyakov 1975, Migdal 1976, Brézin and Zinn-Justin 1976a, b, Brézin et al 1976a, b, Nelson and Pelcovits 1976, 1977 , Hikami and Brézin 1978) to produce $\epsilon$ expansions for Heisenberg-like systems in $2+\epsilon$ dimensions.

The purpose of this paper is to identify, for systems with a discrete symmetry, the Goldstone modes which play the roles described above for systems with a continuous symmetry. The key to this programme rests in recognising that in systems with a discrete symmetry a crucial role is played for $T<T_{\mathrm{c}}$ by the surfaces separating the two (or more) discrete phases, such as the surface of a critical droplet in the metastable phase, or the planar interface between two phases. Now the positions of these surfaces automatically break the Euclidean invariance of the original theory and fluctuations in the position of these surfaces can be regarded as the Goldstone modes of the spontaneously broken Euclidean invariance. Thus we are led to write down the effective Hamiltonians describing these fluctuations, in which the full Euclidean invariance is realised non-linearly.

In the next section we review how the Goldstone modes arise in a field theory calculation and write down the effective Hamiltonians for these modes. We conclude in $\S 3$ by discussing the significance of these models for the essential singularity at a first-order phase transition. The appendix contains explicit calculations of the singularity structure in $d$ dimensions.

\section{The non-linear models}

Our starting point is the conventional Landau-Ginzburg-Wilson Hamiltonian for a one-component real field $\phi(x)$ :

$$
\mathscr{H}=\int \mathrm{d}^{d} x\left[\frac{1}{2}(\nabla \phi)^{2}-\frac{1}{2} \mu^{2} \phi^{2}+\frac{1}{4} g \phi^{4}-H \phi\right] .
$$

The negative mass term $\left(-\mu^{2}\right)$ gives a double well potential describing an Ising-like system below its critical temperature. We are interested in small or zero external field $H$. The dimension of space is denoted by $d$. The partition function, free energy and correlation functions are obtained by the functional integral over fields $\phi$ :

$$
\begin{aligned}
& Z=\exp -\mathscr{F}=\int \mathscr{D} \phi \exp -\mathscr{H} \\
& \left\langle\phi\left(x_{1}\right) \ldots \phi\left(x_{n}\right)\right\rangle=Z^{-1} \int \mathscr{D} \phi \phi\left(x_{1}\right) \ldots \phi\left(x_{n}\right) \exp -\mathscr{H} .
\end{aligned}
$$

The simplest problem in which the Goldstone modes appear is that of the planar interface between two phases $(\langle\phi\rangle>0$ and $\langle\phi\rangle\langle 0)$ when $H=0$ (see the recent articles by Ohta and Kawasaki (1977), Rudnick and Jasnow (1978) and Jasnow and Rudnick (1978) and references therein). This is described by the familiar one-dimensional solution of the double potential well

$$
\phi_{c}(x)=\frac{\mu}{\sqrt{g}} \tanh \left(\frac{\mu}{\sqrt{2}}\left(z-z_{0}\right)\right)
$$


where $z$ denotes for convenience the $d$ th coordinate. The interface can have any position $z_{0}$ and is of thickness $\mu^{-1}$. Given this classical solution we evaluate the effect of fluctuations by writing

$$
\phi=\phi_{c}+\hat{\phi} \text {. }
$$

Expanding for $\hat{\phi}$ small we obtain a new perturbation expansion

$$
Z=\exp \left(-\mathscr{H}\left(\phi_{c}\right)\right) \int \mathscr{D} \hat{\phi} \exp -\left(\frac{i}{2} \int \hat{\phi} \mathscr{M} \hat{\phi}+O\left(\hat{\phi}^{3}\right)\right)
$$

where

$$
M=-\nabla^{2}-\mu^{2}+3 g \phi_{\mathrm{c}}^{2}(z)
$$

The nature of this perturbation expansion is controlled by the spectrum of $\mathscr{M}$. Firstly, it is straightforward to show that

$$
\psi_{0}(x)=\frac{\partial}{\partial z_{0}} \phi_{c}(x)=-\frac{\mu^{2}}{\sqrt{2 g}} \operatorname{sech}^{2}\left(\frac{\mu}{\sqrt{2}}\left(z-z_{0}\right)\right)
$$

is an eigenfunction of $\mathscr{M}$ with zero eigenvalue; it is the Goldstone mode associated with the spontaneous breaking of transiation invariance by $\phi_{\mathrm{c}}(x)$ and the eigenfunction (6) just corresponds to infinitesimal translation of the interface. More importantly for our purpose, the eigenfunctions $\psi_{\boldsymbol{q}}(\boldsymbol{\rho}, z)=\exp (\mathrm{i} \boldsymbol{q} \cdot \boldsymbol{\rho}) \psi_{0}(z)$, where $\boldsymbol{\rho}$ denotes the $(d-1)$ coordinates transverse to $z$, have eigenvalues $q^{2}$; they represent fluctuations of the interface away from the plane and can be regarded as the excitations of the zero-mode $\psi_{0}(z)$. Following the earlier arguments, the interface is therefore a critical problem with a massless field depending on the transverse coordinates $\rho$ and our first aim is to obtain the effective interaction amongst these modes to see if higher-order terms are likely to be important because of infrared singularities.

In group theoretical terms we are required to find the appropriate Hamiltonian which is a function of a field $f(\boldsymbol{\rho})$ and which is invariant under the Euclidean group in $d$ dimensions realised by non-linear transformations on the field $f$. This is a problem with a standard solution in the case of spontaneously broken internal symmetries (Weinberg 1968, Coleman et al 1969, Callan et al 1969, Meetz 1969, Isham 1969, Barnes et al 1972). In our case we are dealing with a spontaneously broken space symmetry, the Euclidean group, for which the general theory of non-linear realisations has, to our knowledge, not been developed. However, there is a very simple geometrical picture which gives the required results, as follows. The energy we are concerned with in these fluctuations is the surface energy. Geometrically the total surface area for a displacement $f(\boldsymbol{\rho})$ away from planar is

$$
S=\int \mathrm{d}^{d-1} \rho\left[1+(\nabla f)^{2}\right]^{1 / 2}
$$

The total surface area is clearly an invariant of the $d$-dimensional Euclidean group, and following the spin-wave approach, we write the reduced Hamiltonian as

$$
\mathscr{H}_{\mathrm{eff}}=\frac{1}{T} \int \mathrm{d}^{d-1} \rho\left[1+(\nabla f)^{2}\right]^{1 / 2}
$$

where $T$ is a measure of the absolute temperature. This is clearly invariant under the Euclidean group of the $(d-1)$ transverse directions; the reader may easily verify that it is also invariant under the non-linear transformations: 
(i) $f \rightarrow f+a$, corresponding to translation by amount $a$ in the $z$ direction, and

(ii) $f \rightarrow f-\theta f \partial_{i} f-\theta \rho_{i}$, corresponding to rotation of the surface by infinitesimal angle $\theta$ in the $\left(\rho_{i}, z\right)$ plane, where $\rho_{i}$ is one of the transverse directions.

Let us now look at the properties of the Hamiltonian (7). Expanding the square root for small $\nabla f$, we pick up the free term $(\nabla f)^{2} / 2$; this is in agreement with the $q^{2}$ spectrum discussed previously. This free term has been used (Buff et al 1965, Davis 1977, Weeks 1977) to demonstrate that $\left\langle f^{2}\right\rangle$, which measures the wandering of the interface from planar, diverges in three dimensions or depends logarithmically on, for example, a gravitational stabilising term. We would suggest that this kind of prediction is analogous to the $H^{-(4-d) / 2}$ singularity in the longitudinal susceptibility discussed previously, and that the intrinsic infrared instability of the theory is determined from the effect of the interaction terms, e.g. $\int \mathrm{d}^{d-1} \rho\left[(\nabla f)^{2}\right]^{2}$ of higher order in $\nabla f$. Now simple dimensional analysis shows that such terms are dimensionless for $d=1$. For $d>1, \mathscr{H}_{\text {eff }}$ in $(7)$ must therefore be expected to be non-renormalisable. Correspondingly, its intrinsic infrared singularities are those of mean field theory for $d>1$. There is no paradox regarding the existence of the logarithmic singularity of $\left\langle f^{2}\right\rangle$ in three dimensions; $\left\langle f^{2}\right\rangle$ is a particular correlation function which happens to be infrared-divergent below three dimensions, just as the longitudinal susceptibility in an isotropic magnet is divergent as $H \rightarrow 0$ below four dimensions. The two- and four-point functions of the field $f$ at non-exceptional external momenta are perfectly infrared finite for $d>1$. The parallel with the non-linear $\sigma$ models of spontaneously broken $\mathrm{O}(n)$ invariance is particularly striking in view of the well-known property that Ising-like models (with short-range interactions) have no phase transitions in less than one dimension.

We turn now to a related problem which is even more intimately connected with the nature of the singularity at a first-order transition, i.e. the contribution of the critical droplet (and fluctuations about it) to the free energy in a metastable phase. The importance of droplet configurations at first-order phase transitions has been recognised for a long time (see Fisher (1967) for a review and Domb (1976) and Binder (1977) for more recent contributions). A significant clarification of the role of the critical droplet in the context of the LGW Hamiltonian (1) was made by Langer (1967). (The equivalent problem of vacuum decay in relativistic quantum field theories is discussed by Voloshin et al (1975), Coleman (1977), Callan and Coleman (1977), Stone (1977), Katz (1978) and Affleck (1979 to be published).) He envisaged taking the free energy of the stable phase (say, $H>0,\langle\phi\rangle>0$ ) and continuing to a metastable phase $H<0$. In order to make a smooth continuation the expectation value of the field $\phi$ must not be allowed to flip. In these circumstances there exists a radially symmetric classical solution $\phi_{\mathrm{c}}(r)$ corresponding to the critical droplet; it has an effective radius $r_{0}$ such that the (first-order) variations of the bulk and surface free energies cancel. This classical solution is not an elementary function, but for very small $H$, the droplet radius $r_{0}$ increases like $H^{-1}$, and the interface between the phases approximates locally the planar interface (2). Hence an approximate form for $\phi_{c}(r)$ is

$$
\phi_{\mathrm{c}} \simeq \frac{1}{2}\left(\phi_{+}+\phi_{-}\right)+\frac{1}{2}\left(\phi_{+}-\phi_{-}\right) \tanh \left(\frac{\mu}{\sqrt{2}}\left(r-r_{0}\right)\right)
$$

where $\phi_{+}$and $\phi_{-}$are the classical minima of $\mathscr{H}$.

The Goldstone modes in this case have already been identified by Langer (1967). There is a $d$-fold degenerate eigenfunction $\nabla \phi_{c}(r)$ with eigenvalue zero; it is the $l=1$ Goldstone mode of the spontaneously broken translation invariance. The 'excitations' 
of this mode are the spherical harmonic excitations with angular momentum $l$; these have eigenvalues

$$
E_{l}=\frac{(l-1)(l+d-1)}{r_{0}^{2}}\left[1+\mathrm{O}\left(l^{2} H^{2}\right)\right]
$$

which are determined directly from the angular momentum barrier $l(l+d-2) / r^{2}$ (in $d$ dimensions) shifted so that $l=1$ is the zero mode. The energy of the lowest mode $l=0$ is negative and this implies that in the context of a steepest descent calculation the critical droplet generates the imaginary part of the free energy in this metastable state, as discussed by Langer (1967).

The important point as far as this paper is concerned is that in the limit $H \rightarrow 0$ of interest, $r_{0} \rightarrow \infty$ and we have a band of Goldstone excitations (9) corresponding to spherical harmonic perturbations of the spherical droplet. Again, there is a simple geometrical picture which enables us to obtain the effective Hamiltonian invariant under a non-linear action of the Euclidean group on the field which is now a function of angles or unit vectors, $f(\boldsymbol{\eta})\left(\boldsymbol{\eta}^{2}=1\right)$. Consider a droplet which deviates from the critical droplet by a field $f(\boldsymbol{\eta})$ defined as shown in figure 1 . Physically there are two contributions to the energy of such a droplet, the bulk free energy, proportional to the volume of the droplet and the external field

$$
\mathscr{H}_{\mathrm{v}}=-\frac{|H|}{d} \int \mathrm{d} \Omega\left(r_{0}+f\right)^{d},
$$

and the surface energy of the interface

$$
\mathscr{H}_{\mathrm{s}}=\int \mathrm{d} \Omega\left(r_{0}+f\right)^{d-1}\left[1+\left(r_{0}+f\right)^{-2}\left(L_{i j} f\right)^{2} / 2\right]^{1 / 2} .
$$

Here $L_{i j}=x_{i} \partial / \partial x_{j}-x_{j} \partial / \partial x_{i}$ is the angular momentum generator of rotation in the $i j$ plane. There are many ways of obtaining the geometrical result (11) for the surface

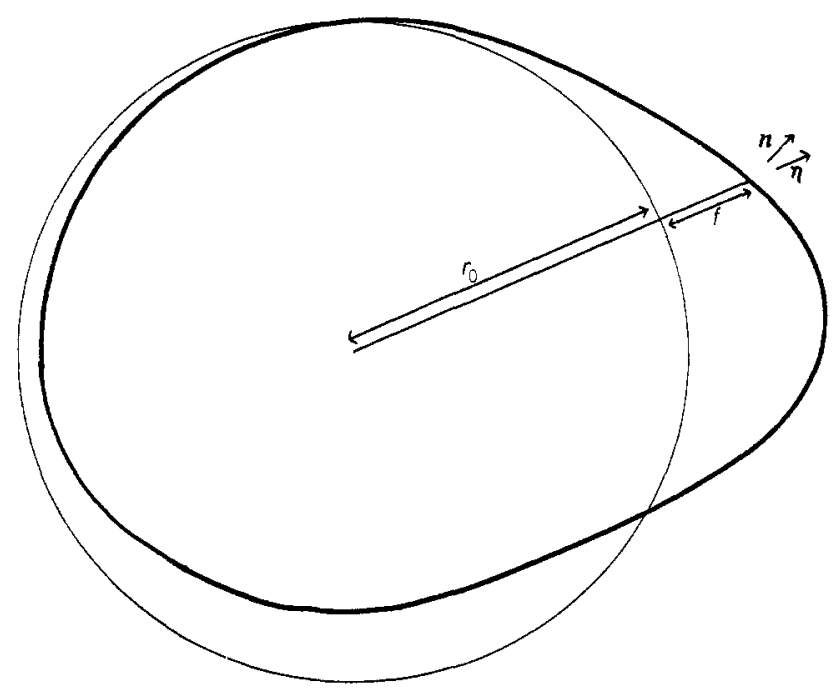

Figure 1. Droplets are described by a field $f(\boldsymbol{\eta})$ which gives the radial displacement from a spherical droplet of radius $r_{0}$. 
area; we found it convenient to generate the normal to the surface

$$
n_{i}(\boldsymbol{\eta})=\left[\left(r_{0}+f\right)^{2}+\left(L_{i j} f\right)^{2} / 2\right]^{-1 / 2}\left[\left(r_{0}+f\right) \eta_{i}-\eta_{k} L_{k i} f\right]
$$

using the neighbouring points $\left(r_{0}+f(\boldsymbol{\eta})\right) \boldsymbol{\eta}$ and $\left(r_{0}+f(\boldsymbol{\eta}+\delta \boldsymbol{\eta})\right)(\boldsymbol{\eta}+\delta \boldsymbol{\eta})$ (where $\delta \eta_{k}=$ $\left.\omega_{i j} L_{i j} \eta_{k} / 2\right)$ which lie on the surface.

The non-linear transformations on the field $f$ under which (10) and (11) are invariant can also be obtained geometrically as shown in figure 2 . The field $f^{\prime}(\boldsymbol{\eta})$ corresponding to the droplet translated by a vector $a$ (the broken line) is obtained by a simple geometrical construction. For infinitesimal $a$ the result is

$$
f^{\prime}(\boldsymbol{\eta})=f(\boldsymbol{\eta})+\boldsymbol{a} \cdot \boldsymbol{\eta}-\frac{a_{i} \eta_{j} L_{i j} f(\boldsymbol{\eta})}{r_{0}+f(\boldsymbol{\eta})} .
$$

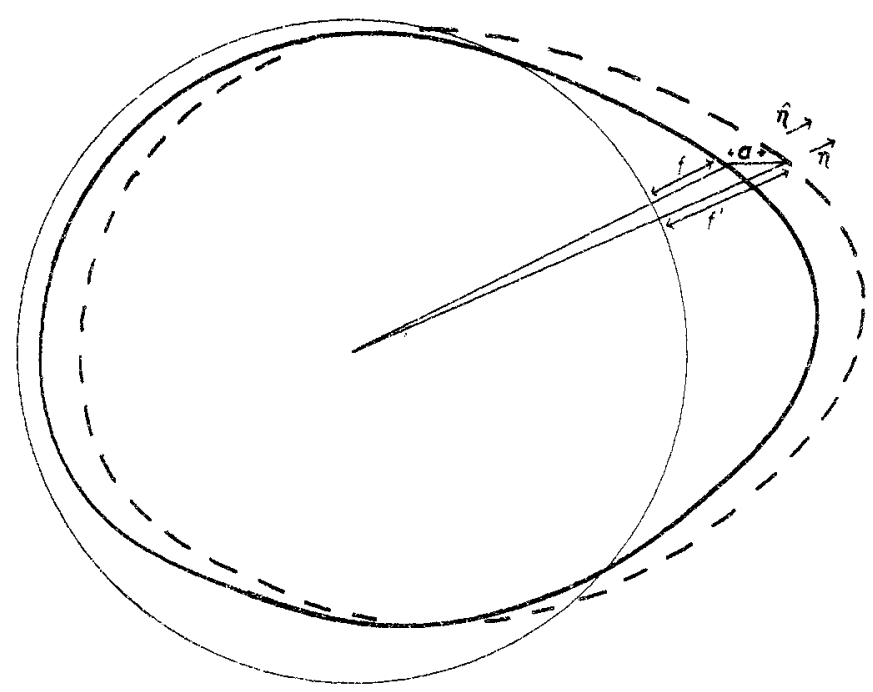

Figure 2. The translated droplet (broken line) is described by a field $f^{\prime}(\eta)$ which can be constructed geometrically as shown.

The last term involving the rotation arises from the fact that the new field $f^{\prime}$ is the translation by $\boldsymbol{a} \cdot \boldsymbol{\eta}$ of the original field $f$ at a rotated point $\hat{\eta}$ as shown in figure 2. The reader may verify directly that expressions (10) and (11) are invariant under the non-linear transformation (12); we remark only that the energy densities are invariant only up to total divergences (involving $L_{i j}$ ) which vanish in the integral $\int \mathrm{d} \Omega$ over all angles.

The full effective Hamiltonian for surface fluctuations of the critical droplet is therefore given by

$$
\mathscr{H}_{\mathrm{eff}}=\mathscr{H}_{\mathrm{v}}+\mathscr{H}_{\mathrm{s}} .
$$

The radius $r_{0}$ is chosen so that we are perturbing about an extremum of $\mathscr{H}_{\mathrm{eff}}$ i.e. $\langle f\rangle=0$. At lowest order in perturbation theory this means ensuring that the term linear in $f$ in (13) vanishes, i.e. $|H|=(d-1) / r_{0}$. Using this value of $H$ we can expand $\mathscr{H}_{\text {eff }}$ up to 
second order in $f$. This yields the free Hamiltonian

$$
\begin{aligned}
\mathscr{H}_{0} & =\frac{1}{2} r_{0}^{d-3} \int \mathrm{d} \Omega\left[\left(L_{i j} f\right)^{2} / 2-(d-1) f^{2}\right] \\
& =\frac{1}{2} r_{0}^{d-3} \int \mathrm{d} \Omega f\left[-L_{i j} L_{i i} / 2-(d-1)\right] f
\end{aligned}
$$

after integration by parts. Now the total angular momentum operator $L_{i j} L_{i j}$ has eigenvalues $-2 l(l+d-2)$ in $d$ dimensions. Hence expression (14) correctly reproduces the spectrum (9), up to a rescaling of $\mathscr{H}$ by $r_{0}^{d-1}$.

Expanding (10) and (11) to higher order to obtain the interaction terms, we see again the appearance of high derivative couplings involving $L_{i j} f$. The ultraviolet divergences come from high angular momentum and are of the same character as those of the planar interface model (7), i.e. they are non-renormalisable in more than one dimension. Indeed, the effective Hamiltonian (13) may be regarded as an infrared regularisation of (1). We tentatively therefore draw the same conclusions as for the planar interface: the Hamiltonian (13) represents the Goldstone modes whose fluctuations destroy the ordering and ensure the absence of a phase transition in systems with a discrete symmetry in less than one dimension.

\section{The first-order phase transition}

It is pertinent to discuss the implications of the above remarks for the singularity at a first-order phase transition. In the appendix we have generalised the calculation of Langer (1967) to obtain the imaginary part of the free energy density in the metastable state in $d$ dimensions. The result of the calculation is

$$
\begin{aligned}
& \operatorname{Im}_{|H| \rightarrow 0} \mathscr{F} \sim A \mu^{d}\left(\frac{\mu^{4-d}}{g}\right)^{d / 2}\left(\frac{\mu^{3}}{|H| \sqrt{g}}\right)^{(d-3) d / 2} \exp \left\{-\frac{\mu^{4-d}}{g}\left[B\left(\frac{\mu^{3}}{|H| \sqrt{g}}\right)^{d-1}+\ldots\right]\right\} \\
& \times\left\{1+\mathrm{O}\left[\left(\frac{\mu^{4-d}}{g}\right)^{-1}\right]\right\} \quad 1<d<5, d \neq 3 .
\end{aligned}
$$

We have written this expression in terms of the dimensionless effective coupling $g / \mu^{4-d}$ and effective field $H \sqrt{g} / \mu^{3} . A$ and $B$ are dimensionless constants. The terms indicated in the exponential are corrections to the leading terms $B\left(\mu^{3} /|H| \sqrt{g}\right)^{d-1}$ of order $H, H^{2}$, etc, in principle. (This is for the general case allowing for an asymmetric double well; we believe that for the $\phi^{4}$ calculation of the appendix these corrections are, in fact, of order $H^{2}, H^{4}$, etc.) The final bracket is to remind us that no explicit calculations have been made beyond some one-loop effects; the $\mathrm{O}\left(\mathrm{g} / \mu^{4-d}\right)$ are certainly important near the critical temperature. The restriction of the result (1.5a) for $d \neq 3$ is explained in the appendix; for $d=3$ the Goldstone excitations produce different logarithmic corrections, which yield for the free energy density

$$
\begin{gathered}
\operatorname{Im}_{|H| \rightarrow 0} \mathscr{F} \sim A \mu^{3}\left(\frac{\mu}{g}\right)^{3 / 2}\left(\frac{\mu^{3}}{|H| \sqrt{g}}\right)^{7 / 3} \exp \left\{-\frac{\mu}{g}\left[B\left(\frac{\mu^{3}}{|H| \sqrt{ } g}\right)^{2}+\ldots\right]\right\} \\
\times\left\{1+\mathrm{O}\left[\left(\frac{\mu}{g}\right)^{-1}\right]\right\} \quad d=3 .
\end{gathered}
$$

This result differs from that quoted in Langer's paper (1967); he quotes the exponent 
$8 / 3$ rather than $7 / 3$. We would like to stress two aspects of the result (15). Firstly, we would remark that the result is likely to be much more universal than one might anticipate. All of the factors are associated simply with the geometrical features of a critical droplet of radius $r_{0} \sim|H|^{-1}$. They do not depend on the specific form of the double well potential. Even the infrared logarithm from the modes (9) is associated with the universal Hamiltonian (13). Further we speculate that the high derivative nature of (13) ensures that there will be no further singularities from the Goldstone modes at higher orders.

The only modification to the result (15) which we can envisage concerns the possibility of a roughening-type transition (Weeks et al 1973, Chui and Weeks 1976, Kosterlitz 1977, Knops 1977, Ohta and Kawasaki 1978). This transition is conventionally considered in the context of a planar interface; models in which the Euclidean invariance is broken by a microscopic length scale can exhibit a transition from interface behaviour described by (7) to a localised interface of finite width as the temperature is lowered through the roughening temperature $T_{R}$. If the analogous phenomenon occurs for the fluctuations in the surface of the critical droplet, then we would expect that the infrared singularities ( $\ln r_{0}$ terms) predicted from the Hamiltonian (13) would be frozen out at temperatures $T<T_{\mathrm{R}}$.

Secondly, if we can assume that the singularities of $\mathscr{F}$ near $H=0$ permit a dispersion relation involving the discontinuity $2 \mathrm{Im} \mathscr{F}$ across the tip of the cut along the $H<0$ axis, then the essential singularity in the free energy for $H>0$ is given by

$$
\mathscr{F}(H)=\frac{1}{\pi} \int_{H^{\prime}<0} \frac{\mathrm{d} H^{\prime} \operatorname{Im} \mathscr{F}\left(H^{\prime}\right)}{H^{\prime}-H} .
$$

In this expression, $\operatorname{Im} \mathscr{F}\left(H^{\prime}\right)$ is given by (15); apart from the Goldstone modes handled as described above, this is a 'one-length-scale calculation' $\left(r_{0} \sim\left|H^{\prime}\right|^{-1}\right)$. We can interpret the dispersion integral over $H^{\prime}$ as a sum over the contribution of 'virtual droplets' of a continuous range of radii-a 'many-length-scale' contribution to $\mathscr{F}$. We believe that the dispersion integral (16) is a good way to understand the 'sum over droplets' responsible for the essential singularity in $\mathscr{F}$ in the stable region. It provides the basis for a droplet model in which the deviations from spherical droplets are precisely controlled by the effective Hamiltonian (13).

In the context of a 'fate of the false vacuum' calculation, we have extended the results of Callan and Coleman (1977) by computing the 'universal' infrared singular part of the functional determinant $\rightarrow$ over small oscillations. Thus we correct the leading power of $\mu^{3} /|H| \sqrt{g}$ in $(15 a)$ from $(d-1) d / 2$ to $(d-3) d / 2$ and in $(15 b)$ from 3 to $7 / 3$.

In conclusion, although there are many gaps in detail in the above picture, we believe it provides new insight into the nature of the singularity at a first-order phase transition and into the disappearance of a phase transition in Ising-like systems as the dimension of space is lowered to 1.

\section{Acknowledgments}

DJW thanks K J Barnes, M E Fisher, J S Langer, D R Nelson, L Schulman and R K P

\footnotetext{
$\uparrow$ After completion of our work, we became aware of the paper by Affleck (1979 to be published) who reports a much more complete calculation of the functional determinant in four dimensions. His method can be extended to $d$ dimensions, and the conclusions support the forms (15) for the singular part of $\operatorname{Im} \mathscr{F}$ as $|H| \rightarrow 0$. We are very grateful to I $\mathrm{K}$ Affleck for illuminating discussions on his work.
} 
Zia for useful discussions and is grateful to the Virginia Polytechnic Institute for hospitality during the completion of this work. DAN thanks D Jasnow, R Griffiths, $T$ Ohta and A Schwartz for discussions. NJG thanks the University of Southampton for the award of a postgraduate studentship. This work was supported in part by the National Science Foundation under grant no PHY75-14073-A01.

\section{Appendix}

In this appendix we review the calculations leading to the imaginary part of the free energy quoted in equations $(15 a)$ and $(15 b)$. The calculations follow very closely those of Langer (1967) but are in the style of the more recent work of Coleman (1977), Callan and Coleman (1977), Katz (1978), Stone (1977) and Affleck (1979 to be published). Our contribution corrects a numerical error in Langer's result for three dimensions, and extends his calculation to $d$ dimensions.

The starting point is the LGW Hamiltonian (1) and the classical solution $\phi_{\mathrm{c}}(x)$ representing the critical droplet and given approximately in (8) for a small external field. We now make the substitution $\phi(x)=\phi_{\mathrm{c}}\left(x-x_{0}\right)+\hat{\phi}$ in the functional integral for the free energy. The coordinate $x_{0}$ just represents the position of the centre of the droplet. We control the translation mode of zero energy by excluding this mode from the 'small oscillations' field $\hat{\phi}$, and integrating exactly over the coordinate $x_{0}$ as a collective coordinate. The result of this substitution is a contribution

$$
\int \mathscr{D} \phi \exp -\mathscr{H} \sim \exp \left[-\mathscr{H}\left(\phi_{\mathrm{c}}\right)\right]\left(\int \mathrm{d}^{d} x\right) \int \mathscr{D} \hat{\phi} J \exp \left(-\frac{1}{2} \int \hat{\phi} \mathscr{M} \hat{\phi}+\mathrm{O}\left(\hat{\phi}^{3}\right)\right) .
$$

$J$ is the Jacobian factor for the change of integration variables, involving the norm of the zero eigenfunction $\partial_{x_{0}} \phi_{\mathrm{c}}$ for each of the $d$ zero modes:

$$
J=\left(\frac{1}{d} \int\left(\nabla \phi_{c}\right)^{2} \mathrm{~d}^{d} x\right)^{d / 2}[1+\mathrm{O}(\hat{\phi})] .
$$

The quadratic form $\mathcal{M}$ is given by

$$
\mathcal{M}=-\nabla^{2}-\mu^{2}+3 g \phi_{\mathrm{c}}^{2}(x)
$$

and translating and using the spherical symmetry of the droplet, we have

$$
\mathcal{M}=-\frac{\mathrm{d}^{2}}{\mathrm{~d} r^{2}}-\frac{d-1}{r} \frac{\mathrm{d}}{\mathrm{d} r}+\frac{l(l+d-2)}{r^{2}}-\mu^{2}+\mu^{2} V(r)
$$

where $V(r)$ comes from $\phi_{\mathrm{c}}^{2}(x)$. We know that $V(r)$ is such that $\mathcal{M}$ has the $d$-fold degenerate mode $\partial_{\nu} \phi_{\mathrm{c}}(x)$. This has exactly zero energy and is given approximately from (8) by $\left(x^{\nu} / r\right) \operatorname{sech}^{2}\left[\mu\left(r-r_{0}\right) / \sqrt{2}\right]$; it is a state bound at radius $r_{0}$, i.e. on the surface of the droplet. This is the mode which enters the Jacobian (A2). The $l$ dependence of the excitations (with approximate wavefunctions $Y_{l}^{m} \operatorname{sech}^{2}\left[\mu\left(r-r_{0}\right) / \sqrt{2}\right]$ ) can be obtained from lowest order in perturbation theory of the term $l(l+d-2) / r^{2}$. Since these states are localised at $r=r_{0}$ we obtain the result (9) for the energy levels $F_{n l}(n=0)$ :

$$
E_{0 l}=\frac{(l-1)(l+d-1)}{r_{0}^{2}}\left[1+\mathrm{O}\left(\mu^{-2} r_{0}^{-2}\right)\right]
$$


The corrections to this result come from higher orders in perturbation theory in, for example, $l(l+d-2) / r^{2}$; they involve intermediate states of energy $\mathrm{O}\left(\mu^{2}\right)$ and hence these corrections are negligible provided $l(l+d-2) /\left(r_{0}^{2} \mu^{2}\right)<1$. These are the only modes whose energy tends to zero for small $H$; the other eigenvalues of $\mathcal{M}$ (consisting in the $\phi^{4}$ theory of a set of rotational excitations of a bound state starting at $E_{1,0}=3 \mu^{2} / 2$ and a band of continuum scattering states starting at $E=2 \mu^{2}$ ) are separated by a gap of order $\mu^{2}$. Thus we need only consider $E_{0 l}$ when evaluating the infrared singularity in the free energy.

The actual quantity we need to calculate is the imaginary part of the free energy $\mathscr{F}$ per unit volume. By a standard argument (Callan and Coleman 1977) we exponentiate the result (A1) to give the contribution of a dilute gas of widely separated critical droplets' to $Z$. Thus the contribution of the droplets to $\bar{F}=\ln Z$ is of the form

$$
\begin{gathered}
\mathscr{F}_{\text {droplets }} \sim \frac{\exp \left[-\mathscr{H}\left(\phi_{c}\right)\right]\left(\int \mathrm{d}^{d} x\right) \int \mathscr{D} \hat{\phi} J \exp \left(-\frac{1}{2} \int \hat{\phi} M \hat{\phi}+\mathrm{O}\left(\hat{\phi}^{3}\right)\right)}{\int \mathscr{D} \phi \exp \left(-\frac{1}{2} \int \phi M_{0} \phi\right)} \\
\sim \exp \left[-\mathscr{H}\left(\phi_{c}\right)\right] J\left(\int \mathrm{d}^{d} x\right)\left(\frac{\operatorname{det}\left(M_{0}\right)}{\operatorname{det}\left(\mathcal{M}^{\prime}\right)}\right)^{1 / 2}
\end{gathered}
$$

where $\operatorname{det} \mathcal{M}^{\prime}$ is the product of eigenvalues of $\mathscr{M}$ with the $d$ zero-energy modes removed, and det $\mathscr{M}_{0}$ is the product of eigenvalues of the quadratic part of $\mathscr{H}$ evaluated about the unstable ground state. The high-energy modes of $\mathscr{M}^{\prime}$ and $\mathscr{M}_{0}$ control the ultraviolet renormalisations of the theory and a detailed computation of the complete functional determinant is needed to fix the scale factor in (A5). We, however, are only interested in the infrared structure of $\mathscr{M}$ and thus need only consider the modes $E_{0}$ from (A4) and the corresponding modes with energy of order $\mu^{2}$ in $M_{0}$. Thus, for our purpose we have

$$
\mathscr{F}_{\text {droplets }} \sim \exp \left[-\mathscr{H}\left(\phi_{c}\right)\right] J\left(\int \mathrm{d}^{d} x\right) \mu^{d} \exp \left\{\frac{1}{2}\left[\sum_{l \neq 1}^{L} \nu_{l} \ln \left(\mu^{2} / E_{0 l}\right)\right]\right\} .
$$

Here

$$
\nu_{l}=\frac{\Gamma(l+d)}{\Gamma(d) \Gamma(l+1)}-\frac{\Gamma(l+d-2)}{\Gamma(d) \Gamma(l-1)} \quad l>1
$$

is the degeneracy of the $d$-dimensional spherical harmonics. The $l=0$ mode in the denominator has negative energy and thus contributes a factor $i$ to the right-hand side, showing that the droplets indeed generate an imaginary part for $\mathscr{F}$. The upper limit $L$ is given by $L^{2} \sim r_{0}^{2} \mu^{2}$ where the approximation (A4) breaks down. We are now in a position to compute the factors in (A6).

\section{A1.1. The classical energy}

We have constructed $\phi_{\mathrm{c}}$ so that it tends to the metastable minimum $\phi_{+}$as $r \rightarrow \infty$. Thus we have $\mathscr{H}\left(\phi_{c}\right)=\mathscr{H}\left(\phi_{+}\right)+$convergent integrals, where $\mathscr{H}\left(\phi_{+}\right)$is just the classical approximation for the free energy in the metastable phase; it contributes additively to the free energy and does not exponentiate in the dilute gas approximation leading to (A5). Neglecting this term, we proceed to evaluate the remaining iniegrals in $\mathscr{H}\left(\phi_{\mathrm{c}}\right)$ for 
small $H$ and large $r_{0}$. The result is

$$
\begin{gathered}
\mathscr{H}\left(\phi_{\mathrm{c}}\right)=\frac{2 \pi^{d / 2}}{\Gamma(d / 2)} \int_{0}^{\infty} \mathrm{d} r r^{d-1}\left[\frac{1}{2}\left(\nabla \phi_{c}\right)^{2}-\frac{1}{2} \mu^{2}\left(\phi_{\mathrm{c}}^{2}-\phi_{+}^{2}\right)+\frac{1}{4} g\left(\phi_{\mathrm{c}}^{4}-\phi_{+}^{4}\right)-H\left(\phi_{\mathrm{c}}-\phi_{+}\right)\right] \\
\simeq \frac{2 \pi^{d / 2}}{\Gamma(d / 2)}\left(\frac{2 \sqrt{2} \mu^{3} r_{0}^{d-1}}{3 g}-\frac{2|H| \mu r_{0}^{d}}{d \sqrt{g}}\right) .
\end{gathered}
$$

There is an extremum of this expression (a functional saddle point in $\mathscr{H}$ ) at

with energy

$$
r_{0}=\frac{\sqrt{2}(d-1)}{3} \frac{\mu^{2}}{|H| \sqrt{ } g}
$$

$$
\mathscr{H}\left(\phi_{\mathrm{c}}\right)=\frac{2 \pi^{d / 2}}{\Gamma(d / 2)} \frac{2 \sqrt{2}}{3 d} \frac{\mu^{3}}{g}\left(\frac{\sqrt{2}(d-1)}{3} \frac{\mu^{2}}{|H| \sqrt{g}}\right)^{d-1} .
$$

The coefficients of this energy and of $r_{0}$ are not universal; they clearly depend on the structure of the $\phi^{4}$ theory. The singularity structure is, however, universal for couplings of the appropriate dimensionality, and takes the form

$$
\mathscr{H}\left(\phi_{c}\right)=B \frac{\mu^{4-d}}{g}\left(\frac{\mu^{3}}{|H| \sqrt{g}}\right)^{d-1} \text {. }
$$

\section{A1.2. The Jacobian}

The Jacobian for any system with a quadratic kinetic part may be evaluated by a simple virial argument (Callan and Coleman 1977). We have

$$
\mathscr{H}=\int \mathrm{d}^{d} x \frac{1}{2}(\nabla \phi)^{2}+\int \mathrm{d}^{d} x V(\phi) .
$$

At $\phi=\phi_{\mathrm{c}}(x)$ we have a saddle point of $\mathscr{H}$ and thus $\mathscr{H}\left[\phi_{\mathrm{c}}(\lambda x)\right]$ has an extremum at $\lambda=1$. Now

$$
\left.\frac{\mathrm{d} \mathscr{H}\left[\phi_{\mathrm{c}}(\lambda x)\right]}{\mathrm{d} \lambda}\right|_{\lambda=1}=(1-d / 2) \int \mathrm{d}^{d} x\left(\nabla \phi_{\mathrm{c}}\right)^{2}-d \int \mathrm{d}^{d} x V\left(\phi_{\mathrm{c}}\right)=0
$$

Thus

$$
\int \mathrm{d}^{d} x\left(\nabla \phi_{\mathrm{c}}\right)^{2}=d \mathscr{H}\left(\phi_{\mathrm{c}}\right)
$$

and the Jacobian is given by

$$
J=\left[\mathscr{H}\left(\phi_{c}\right)\right]^{d / 2} \propto\left(\frac{\mu^{3}}{|H| \sqrt{ } g}\right)^{(d-1) d / 2}\left(\frac{\mu^{4-d}}{g}\right)^{d / 2} .
$$

\section{A1.3. The spectrum of Goldstone excitations}

We seek to compute the singularity structure of

$$
\begin{aligned}
& \mu^{d} \exp \left(\frac{1}{2} \sum_{l \neq 1}^{L} \nu_{l} \ln \left(\mu^{2} / E_{0 l}\right)\right) \\
& \quad=\frac{i r_{0} \mu^{d+1}}{\sqrt{(d-1)}} \exp \left(-\frac{1}{2} \sum_{l=2}^{L} \nu_{l} \ln \frac{(l-1)(l+d-1)}{r_{0}^{2} \mu^{2}}\right)
\end{aligned}
$$


with upper limit $L=D r_{0} \mu, D \sim 1$. On inserting the expression for $\nu_{l}$, we find

$$
\begin{aligned}
\Gamma(d) \sum_{l=2}^{L} \nu_{l} \ln & \frac{(l-1)(l+d-1)}{r_{0}^{2} \mu^{2}} \\
= & \left.\frac{\Gamma(L+d)}{\Gamma(L+1)}+\frac{\Gamma(L+d+1)}{\Gamma(L)}\right) 2 \ln D\left[1+\mathrm{O}\left(L^{-1}\right)\right] \\
& +2(d+1) \Gamma(d) \ln \left(r_{0} \mu\right)+\sum_{l=2}^{L} \Gamma(l+d) \\
& \times\left(\frac{-4}{\Gamma(l+2)}+\frac{2(d-2)}{\Gamma(l+3)}+\frac{\left(-2 d^{2}+6 d-28 / 3\right)}{\Gamma(l+4)}+\frac{2(d-4)\left(d^{2}-2 d+4\right)}{\Gamma(l+5)}\right) \\
& + \text { pieces finite as } L \rightarrow \infty \text { for } d<5 .
\end{aligned}
$$

The parts of this sum which behave as powers of $r_{0}$ have no universal significance, for they are adjusted or even completely cancelled by ultraviolet renormalisations. The $\ln \left(r_{0} \mu\right)$ part, on the other hand, is not expected to be affected by short distance structure, and enters as a correction to the leading power of $\mu^{3} / H \sqrt{g}$ in (15). Apart from the term in (A11) explicitly containing a logarithm, the only other source of logarithms is a term in the sum tending to a harmonic series $\left(l^{-1}\right)$, in some integer dimension. For $d=1$, the calculation makes no sense, while for $d=2$ or 4 the appropriate term has zero coefficient. Thus we need only consider $d=3$, where we have

$$
-\frac{28}{3} \sum_{l=2}^{L} \frac{\Gamma(l+3)}{\Gamma(l+4)}=-\frac{28}{3} \sum_{l=2}^{L}(l+3)^{-1} \sim-\frac{28}{3} \ln L \quad \text { for large } L .
$$

Thus our result is

$$
\begin{aligned}
\mu^{d} \exp \left\{\frac{1}{2}\left[\sum_{l \neq 1}^{L} \nu_{l} \ln \left(\mu^{2} / E_{0 l}\right)\right]\right\} & \begin{cases}\mathrm{i} \mu^{d}\left(\frac{\mu^{3}}{|H| \sqrt{ } g}\right)^{-d} \exp \left[\langle\text { constant }\rangle\left(\frac{\mu^{3}}{|H| \sqrt{ } g}\right)^{d-1}\right] & d \neq 3 \\
\mathrm{i} \mu^{3}\left(\frac{\mu^{3}}{|H| \sqrt{ } g}\right)^{-2 / 3} \exp \left[\langle\text { constant }\rangle\left(\frac{\mu^{3}}{|H| \sqrt{ } g}\right)^{2}\right] & d=3 .\end{cases}
\end{aligned}
$$

The reader will note that the form of this singularity is independent of the exact choice of $D$, as it arises from modes with $l \ll L$.

We finally assemble these results (A8), (A9) and (A12) according to (A5) to give (15) as our final result for the imaginary part of $\mathscr{F}$ per unit volume.

\section{References}

Barnes K J, Dondi P H and Sarkar S C 1972 Proc. R. Soc. A 330389

Binder K 1977 Phys. Rev. B 154425

Brézin E and Zinn-Justin J 1976a Phys. Rev. Lett. 36691

-_ 1976b Phys. Rev. B 143110

Brézin E, Zinn-Justin J and Le Guillou J C 1976a Phys. Rev. D 142615

- 1976b Phys. Rev. B 144976

Buff F P, Lovett R A and Stillinger F H 1965 Phys. Rev. Lett. 15621

Burton W K and Cabrera N 1949 Disc. Faraday Soc. 533 
Callan C G and Coleman S 1977 Phys, Rev. D 161762

Callan C G, Coleman S, Wess J and Zumino B 1969 Phys. Rev. 1772247

Chui S T and Weeks J D 1976 Phys. Rev. B 144978

Coleman S 1973 Commun. Math. Phys. 31259

— 1977 Phys. Rev. D 152929

Coleman S, Wess J and Zumino B 1969 Phys. Rev. 1772239

Davis H T 1977 J. Chem. Phys. 673636

Domb C 1976 J. Phys. A: Math. Gen. 9283

Fisher M E 1967 Physics 3255

Hikami S and Brézin E 1978 J. Phys. A: Math. Gen. 111141

Hohenberg P C 1967 Phys. Rev. 158383

Holstein T and Primakoff H 1940 Phys. Rev. 581098

Horner H and Schäfer L 1978 Z. Phys. 29251

Isham C J 1969 Nuovo Cim. A 61188

Jasnow D and Rudnick J 1978 Phys. Rev. Lett. 41698

Katz H J 1978 Phys. Rev. D 171056

Knops H J F 1977 Phys. Rev. Lett. 39766

Kosterlitz J M 1977 J. Phys. C: Solid St. Phys. 103735

Langer J S 1967 Ann. Phys., NY 41108

Meetz K 1969 J. Math. Phys. 10589

Mermin N D and Wagner H 1966 Phys. Rev. Lett. 171133

Migdal A A 1976 Sov. Phys.-JETP 42743

Nelson D R and Pelcovits R A 1976 Phys. Lett. 57A 23

- 1977 Phys. Rev. B 162191

Ohta T and Kawasaki K 1977 Prog. Theor. Phys. 58467 1978 Prog. Theor. Phys. 60365

Polyakov A M 1975 Phys. Lett. 59B 79

Rudnick J and Jasnow D 1978 Phys. Rev. B 171351

Stone M 1977 Phys. Lett. 67B 186

Voloshin M B, Kobzarev I Yu and Okun L B 1975 Sov. J. Nucl. Phys. 20644

Wallace D J and Zia R K P 1975 Phys. Rev. B 125340

Weeks J D 1977 J. Chem. Phys. 673106

Weeks J D, Gilmer G H and Leamy H J 1973 Phys. Rev. Lett. 31549

Weinberg S 1968 Phys. Rev. 1661568 